\title{
Functional and Morphological Adaptation in DNA Protocells via Signal-Processing Prompted by Artificial Metalloenzymes
}

Avik Samanta, ${ }^{1-4}$ Valerio Sabatino, ${ }^{5}$ Thomas R. Ward, ${ }^{5 *}$ Andreas Walther ${ }^{1-4}$ *

\footnotetext{
${ }^{1}$ A $^{3}$ BMS Lab, Institute for Macromolecular Chemistry, University of Freiburg, Stefan-Meier-Straße 31, 79104 Freiburg, Germany

2 DFG Cluster of Excellence "Living, Adaptive and Energy-Autonomous Materials Systems" (livMatS)@FIT, 79104 Freiburg, Germany.

${ }^{3}$ Freiburg Materials Research Center, University of Freiburg, Stefan-Meier-Straße 21, 79104 Freiburg, Germany

${ }^{4}$ Freiburg Center for Interactive Materials and Bioinspired Technologies (FIT), University of Freiburg, GeorgesKöhler-Allee 105, 79110 Freiburg, Germany

${ }^{5}$ Department of Chemistry, University of Basel, BPR 1096, Mattenstrasse 24a, Biopark Rosental, 4058 Basel, Switzerland

Email: andreas.walther@makro.uni-freiburg.de; thomas.ward@unibas.ch
} 
For life to emerge, confinement of catalytic reactions within protocellular environments has been proposed as a decisive aspect to regulate chemical activity in space. ${ }^{1,2}$ Today, cells and organisms adapt to signals ${ }^{3-7}$ by processing them through reaction networks that ultimately provide downstream functional responses and structural morphogenesis. ${ }^{8,9}$ Re-enacting such signal processing in de-novo designed protocells is a profound challenge, but of high importance for understanding the design of adaptive systems with life-like traits. We report on engineered all-DNA protocells ${ }^{10}$ harboring an artificial metalloenzyme ${ }^{11}$ whose olefin metathesis activity leads to downstream morphogenetic protocellular responses with varying levels of complexity. The artificial metalloenzyme catalyzes the uncaging of a pro-fluorescent signal molecule, that generates a self-reporting fluorescent metabolite designed to weaken DNA duplex interactions. This leads to pronounced growth, intra-particular functional adaptation in the presence of a fluorescent DNA mechanosensor, ${ }^{12}$ or inter-particle protocell fusion. Such processes mimic chemically transduced processes found in cell adaptation and cell-to-cell adhesion. Our concept showcases new opportunities to study lifelike behavior via abiotic bioorthogonal chemical and mechanical transformations in synthetic protocells. Furthermore, it reveals a strategy for inducing complex behavior in adaptive- and communicating softmatter microsystems, and illustrates how dynamic properties can be upregulated and sustained in microcompartmentalized media.

Living cells are highly inspiring for their unique ability to perform complex tasks, such as division, differentiation and tissue formation via internal signal processing and intercellular communication. ${ }^{6,13}$ In cells, a diversity of different signals are processed in a crowded environment using reaction networks, that may even be organized spatially in liquid/liquid phase-segregated membraneless organelles. ${ }^{14-16}$ Protocell models resulting from the self-assembly of amphiphiles, such as phospholipid liposomes, have been used to investigate prebiotic compartmentalization and primitive processes, such as catalysis, metabolism, and replication. ${ }^{17-20}$ These however, seldom recapitulate some of the critical features that are essential to cellular function: macromolecular crowding and phase-segregation. ${ }^{21}$ To this end, (bio)polymer coacervates have been suggested as model systems, and recent studies have reported gene expression, ${ }^{15}$ ribonucleic acid catalysis ${ }^{22}$ and multienzyme iterative processing in multicomponent microdroplets. ${ }^{23,24}$ Some of the most complex mimics may include DNA-containing protocells that can exchange DNA as information and may allow for simple communication. ${ }^{25,26}$ However, it is of critical importance to develop strategies able to convert signals from diverse origin to allow for intra- or inter-protocell downstream processes such as functional adaptation, communication and simple morphogenesis which may, in the long term, allow for the development of prototissues. $^{27}$

Artificial metalloenzymes (ArMs) are an emerging tool for advanced biohybrid catalysis containing an organometallic moiety as synthetic co-factor embedded in a protein scaffold. They combine features of homogeneous and enzymatic catalysts. ArMs can be evolved by genetic means, and hold great promise to impart new types of abiotic activities in biological systems. ${ }^{28,29}$ We have developed versatile ArMs based on 
the biotin-streptavidin (Sav) technology, that have been shown to operate in cells and allow for spatiallycontrolled bioorthogonal activation of functional molecules such as prodrugs. ${ }^{11,30}$ Other bioorthogonal uncaging strategies have been reported for the on-demand release of cargoes under physiological conditions. ${ }^{31,32,33}$ This may lead to a biological response resulting from an abiotic catalyst.

Inspired by such conversion strategies foreign to the host system, we set out to compartmentalize an artificial metathase based on the biotin-Sav technology inside an all-DNA protocell. This allows to investigate the effect of (bio)molecular crowding on catalytic performance. More importantly, it introduces new-to-nature catalytic activities, beyond the commonly used DNA-tethered enzymes ${ }^{34}$ or DNAzymes, allowing processing of non-DNA signals within DNA nanoscience environments, and enabling access for downstream reactions using non-DNA metabolites. For this purpose, we rely on a "close-to-release" reaction cascade that leads to a "gain-offunction" via ring-closing metathesis (RCM)-triggered uncaging of a pro-fluorescent signal. The uncaged product is reactive as a downstream metabolite by interacting with duplex DNA. This strategy allows to (i) monitor the compartmentalized abiotic reaction and (ii) modulate signal transduction, which leads to downstream morphological responses (Fig. 1). We show that the accumulated product can dynamize DNA duplexes, ultimately resulting in protocellular growth, mechanical activation, adhesion and fusion, thus mimicking rudimentary, functional and morphological adaptation of (proto)-cells.

Building on our recent work ${ }^{10}$, we prepared all-DNA based protocells (PCs) with liquid ssDNA interior and DNA hydrogel shells to compartmentalize the ArMs. The PCs are formed by a simple self-compartmentalization during a temperature ramp (ca. $10 \mathrm{~min}$ ) of aqueous systems of two ssDNA multiblock copolymers, $\mathrm{p}\left(\mathrm{A}_{20}-\mathrm{m}\right)$ and $p\left(T_{20}-n\right)$ (Supplementary Fig. S1, ca. 15-55 repeats of the blocks). $A_{20}$ and $T_{20}$ represent homo-repeats of 20 adenine and 20 thymine nucleobases, while $\mathrm{m}$ and $\mathrm{n}$ stand for defined barcode sequences for functionalization (Fig. 1). In short, heating leads to $A_{20} / T_{20}$ duplex dissociation and $p\left(A_{20}-m\right)$ undergoes liquid/liquid phase-segregation, while $p\left(T_{20}-n\right)$ remains dissolved in solution. During cooling, re-hybridization of $A_{20} / T_{20}$ occurs quickly at the periphery of the $p\left(A_{20}-m\right)$ phase-segregated droplets, leading to a hydrogel shell stabilized by $A_{20} / T_{20}$ duplex. Subsequently, the $p\left(A_{20}-m\right)$ dissolves, but remains entrapped in a liquid state under high osmotic pressure and macromolecular crowding in the tight hydrogel-like shell. In addition to their straightforward assembly, such PCs allow selective functionalization of the core and the shell using the barcodes $(m, n)$.

Immobilization of the ArM is achieved by functionalizing the $\mathrm{m}$ core barcodes with a complementary biotinylated Biot-m* ssDNA, that binds to Sav. Thanks to its homotetrameric nature, immobilized Sav can additionally accommodate a biotinylated Hoveyda-Grubbs catalyst (Biot-Ru; Supplementary Fig. S2). To ensure efficient immobilization, the molar ratio of Sav to Biot- $\mathrm{m}^{*}$ was set to $1: 1$, providing, on average, $75 \%$ free binding sites for reaction with Biot-Ru. An appealing feature of such ArMs is the possibility to improve their catalytic performance via site-directed mutagenesis (Supplementary Note 1 ). ${ }^{11}$ We surmised that this strategy 
might provide a versatile tool for the optimization of catalysts within the crowded environment provided by the DNA PCs.
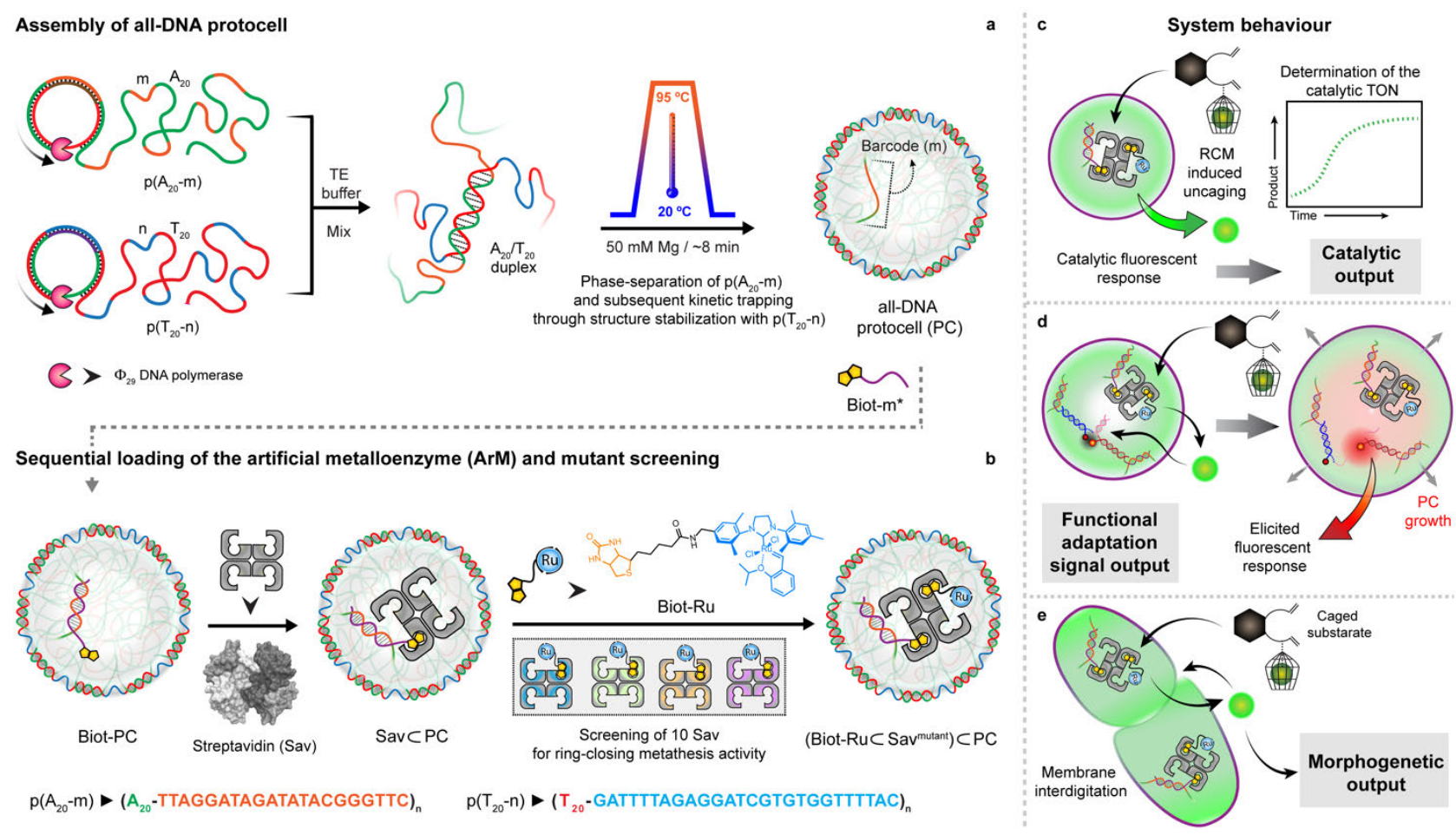

Fig. 1 | Design concept, strategy, and system behaviour of artificial metalloenzyme (ArM)-catalyzed signal conversion and downstream adaptation inside all-DNA protocells (PCs). (a) Synthesis of sequence-controlled multiblock ssDNA polymers via rolling circle amplification (RCA). The mixture of the ssDNA polymers is subjected to a heating ramp ( 3 $\left.{ }^{\circ} \mathrm{C} / \mathrm{min}\right)$ in the presence of $\mathrm{Mg}^{2+}$. Thermally-induced phase-separation of $\mathrm{p}\left(\mathrm{A}_{20}-\mathrm{m}\right)$ during heating and duplex $\left(\mathrm{A}_{20} / \mathrm{T}_{20}\right)$ formation (at the coacervate surface) during cooling, leads to the self-assembly of kinetically-trapped all-DNA PCs. The core of the $P C s$ is composed of liquid $p\left(A_{20}-m\right)$ while the shell is constituted with $p\left(T_{20}-n\right)$ crosslinked via $A_{20} / T_{20}$ duplexes. (b) Sequential loading and assembly of the artificial metalloenzyme (ArM) is achieved by attaching Biot- $\mathrm{m}^{*}$ to the core barcode $\mathrm{m}$, followed by addition of streptavidin (Sav) and a biotinylated olefin metathesis catalyst (Biot-Ru), to afford a

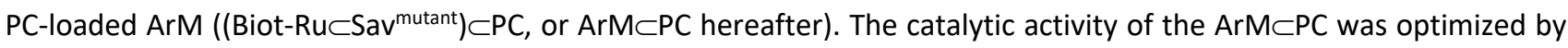
screening 10 Sav mutants. (c-e) Schematic representation of three adaptive responses of the RCM inside the PCs. (c) The immobilized ArM catalyzes a DNA-orthogonal uncaging reaction giving rise to a primary fluorescent signal. (d) The uncaged product instigates swelling of the PCs and destabilizes a mechanofluorescent force module (installed in PCs) which, triggers a secondary fluorescence output. (e) The accumulated uncaged product also induces membrane dynamization of the PCs, resulting in pronounced morphological transformations, ultimately leading to PC fusion.

First, we demonstrate the uptake and selective functionalization of the individual compartments using fluorescent model compounds: (i) Atto 647 - $\mathrm{n} *$ (red channel) for the PC shell, (ii) Oregon-green 488 labeled Sav$\mathrm{OG}_{488}$ (green channel) and (iii) biotinylated-Atto ${ }_{565}$ (magenta channel) as a catalyst mimic (Fig. 2a,b). After functionalization of the PC core barcodes $(m)$ with Biot-m*, Sav- $\mathrm{OG}_{488}$ - that binds to Biot-m* - and the catalyst-mimic (Biot-Atto ${ }_{565}$ ) - that fills the remaining biotin-binding sites of the immobilized Sav- $\mathrm{OG}_{488}-$ were added sequentially. The loading protocol, adjuvants and stoichiometry were fine-tuned to ensure a homogeneous loading of the PCs. Confocal laser scanning microscopy (CLSM) of the loaded PCs reveals their spatially programmed assembly by visualizing their respective fluorescent signals. This confirms that the PC shells are sufficiently porous to allow uptaking Sav, the largest entity in our functionalization scheme (ca. 5·5·5 $\mathrm{nm}^{3}$ ). 


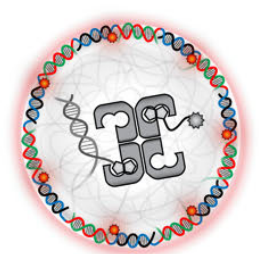

b
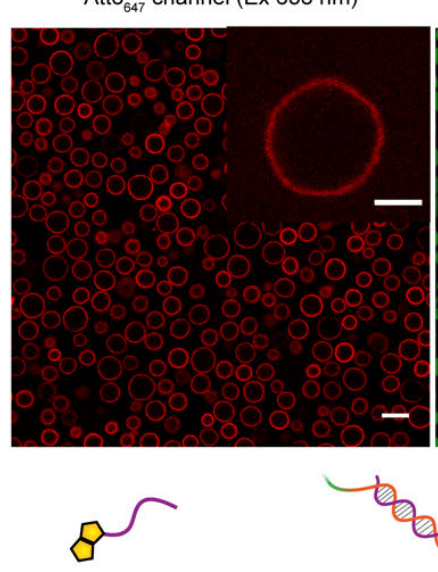

Biot-m*

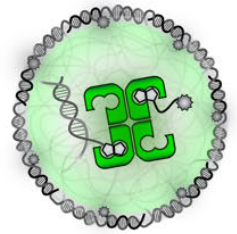

Oregon Green $_{488}$ channel (Ex $\left.488 \mathrm{~nm}\right)$

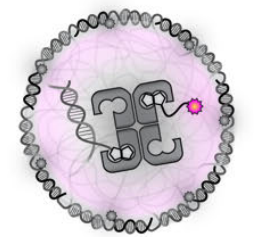

Atto $_{565}$ channel (Ex $552 \mathrm{~nm}$ )

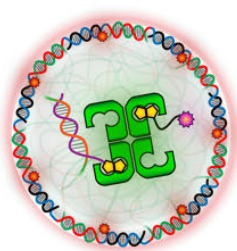

Merged channels
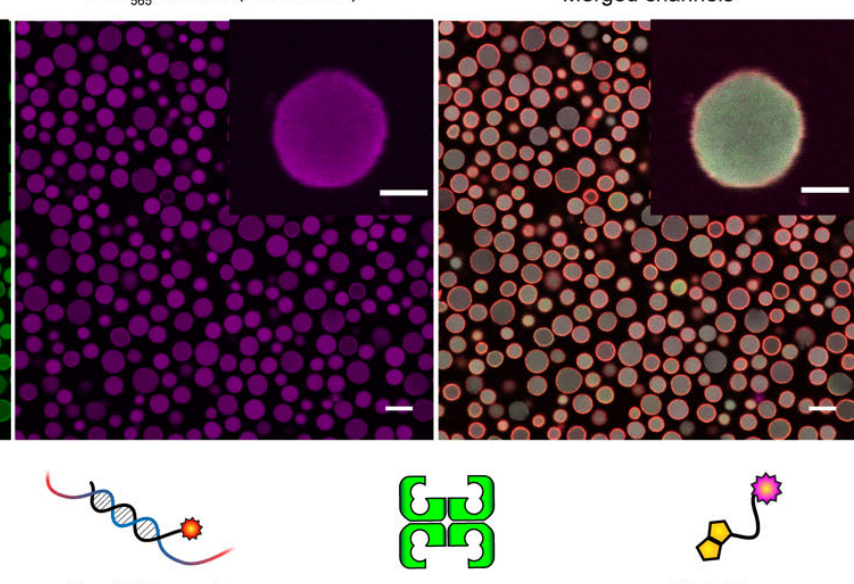

$p\left(T_{20}-n\right) / A t_{t o}{ }_{647}-n^{*}$ duplex

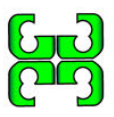

Sav- $\mathrm{OG}_{488}$

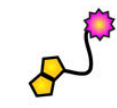

Biot-Atto ${ }_{565}$ (Catalyst-mimic)
C PC: Liquid core

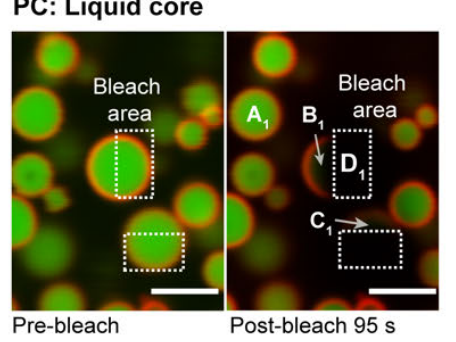

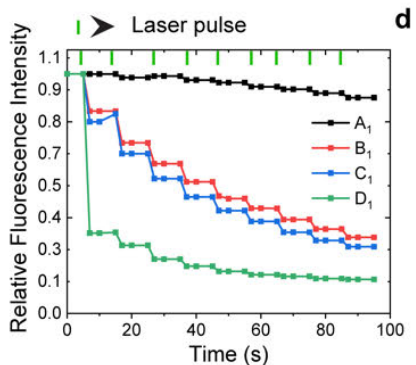

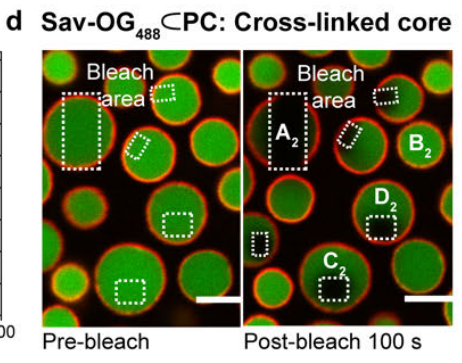

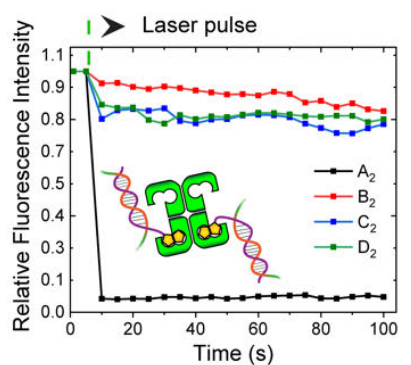

Fig. 2 |Compartment-selective functionalization of PCs (a) Schematic representation of (Biot-Atto ${ }_{565} \subset$ Sav) $\subset$ PC depicting all of its components. (b) CLSM images of (Biot-Atto ${ }_{565} \subset$ Sav) $\subset$ PC: The shell of the PCs is functionalized with Atto ${ }_{647}-n^{*}$ (red channel). The Sav- $\mathrm{OG}_{488}$ (green channel) is immobilized in the PC core via Biot-m* (hybridized with barcodes $\mathrm{m}$ ), followed by biotin-Atto 565 (magenta channel) binding to the Sav-OG ${ }_{488}$. (c) CLSM images of pristine PCs before and after photo-bleaching within the dotted rectangles. The corresponding fluorescence intensities at positions $A_{1}, B_{1}, C_{1}$, and $D_{1}$ (during nine sequential photo-bleaching events) reveal that the core is liquid ( $B_{1}, C_{1}$ partially bleached) and that the shells are crosslinked (i.e., only bleached in the irradiated areas). (d) CLSM images of Sav-loaded PCs before and after photobleaching in the dotted rectangles. The corresponding fluorescence during photobleaching at position $A_{2}, B_{2}, C_{2}$, and $D_{2}$ highlight the gelled core, due to the multivalent crosslinking of the Sav-OG ${ }_{488}$ with Biot-m* (hybridized to the $p\left(A_{20}-m\right)$ polymer). The green ticks on the top indicate the photo-bleaching events. Scale bars: $3 \mu \mathrm{m}$.

The physical state of the pristine and Sav-loaded (Sav $\subset$ PCs) PCs was elucidated by fluorescence recovery after photobleaching (FRAP; Fig. 2c,d, Supplementary movies S1 and S2). The pristine PCs exhibit near-simultaneous bleaching of both the irradiated and non-irradiated core areas within single PCs (areas $B_{1}, C_{1}$ and $D_{1}$, Fig. 2c). Repeated photobleaching leads to stepwise bleaching of the full PC core, confirming a liquid-like interior of the PCs surrounded by a hydrogel-like shell. This agrees with our earlier report highlighting a liquid-like interior and quick reorganization. ${ }^{10}$ In contrast, the Sav- $\mathrm{OG}_{488} \subset \mathrm{PC}$ cores and shells show almost no fluorescence recovery within the exposed area after a single pulse of photo-bleaching (area $A_{2}$ in Fig. 2d). Although the nonirradiated areas lose $20 \%$ of their initial intensity after bleaching, a complete reorganization and 
homogenization of the interior does not occur. This observation suggests that the Sav serves as a multivalent crosslinker of the $p\left(A_{20}-m\right) / B i o t-m *$ interior. The interior is in a gel state.

Next, we incorporated the metathase (Biot-RuCSav) in the PC core and monitored the catalytic RCM reaction and the signal translation resulting from the uncaging of the pro-fluorescent cargos. We evaluated two dienecontaining substrates which display self-reporting functions and from which we expected a downstream metabolite interaction with DNA: (i) a naphthalene precursor which releases umbelliferone (Subs-I), and (ii) a dimethoxynaphthalene precursor (Subs-II) which spontaneously eliminates water upon RCM (Fig. 3a, f). Uncaged umbelliferone and dimethoxynaphthalene allow monitoring the RCM activity by fluorescence spectroscopy and GC-MS, respectively. Importantly, with respect to downstream response, coumarinderivatives, such as umbelliferone, are well-known intercalators of dsDNA (in particular $A / T$ ). ${ }^{35}$ We further hypothesized that dimethoxynaphthalene might also interact with DNA due to its hydrophobic nature and the ability for $\pi$-stacking. ${ }^{36}$

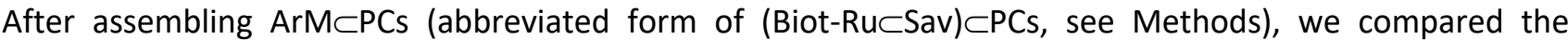
turnover numbers of the ArM (TONRu) in solution and encapsulated state (in the PC interior) for both Subs-I and Subs-II (Fig. 3c,g). The TON $\mathrm{RuS}_{\mathrm{S}}$ in PCs are normalized to the Biot-Ru content determined by inductively coupled plasma mass spectrometry (ICP-MS) and to purified PCs. We find that for Subs-I and Subs-II, the $\mathrm{TON}_{\mathrm{Ru}} \mathrm{S}$ of $\mathrm{ArM} \subset \mathrm{PC}$ are 2-fold and 3-fold higher respectively (in case of wild type Sav ${ }^{\mathrm{WT}}$ ) than the free $\operatorname{ArM}$ in solution. Next, we screened a focused library of 10 Sav mutants, identified in previous optimization

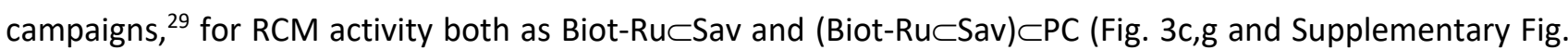

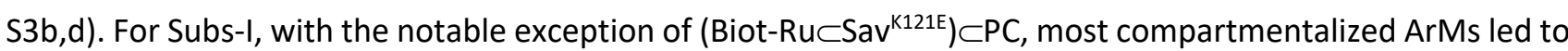

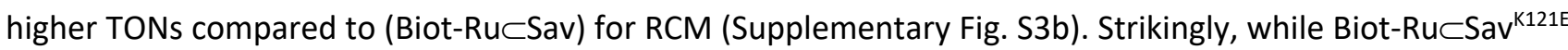
was the most active mutant screened in solution, (Biot-RuCSav $\left.{ }^{\mathrm{K} 121 \mathrm{E}}\right) \subset \mathrm{PC}$ was the least active in the PC. Introduction of an additional mutation, thus relocating a cationic lysine residue from position 121 to 118 , afforded the most active compartmentalized $\operatorname{ArM}\left(\right.$ Biot-RuCSav $\left.^{\mathrm{N118K}}{ }^{\mathrm{K} 121 \mathrm{E}}\right) \subset \mathrm{PC}$ and the least active $\operatorname{ArM}$ in

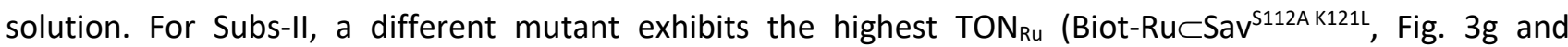
Supplementary Fig. S3d). While challenging to rationalize, these results highlight how subtle changes in the second coordination sphere around the cofactor (i.e. mutation and/or crowding) dramatically affect the RCM activity.

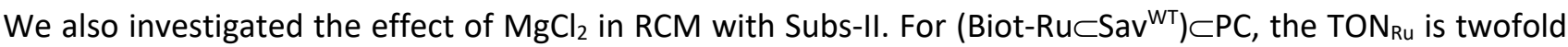
higher in the presence of $200 \mathrm{mM} \mathrm{MgCl}_{2}$ compared to $50 \mathrm{mM} \mathrm{MgCl}_{2}$ (Supplementary Fig. S3e). Higher [ $\mathrm{Cl}^{-}$] have been shown to stabilize the Ru catalyst under physiological conditions, ${ }^{33}$ which is also confirmed in this protocellular environment. Accordingly, we used $200 \mathrm{mM} \mathrm{MgCl}_{2}$ consistently for all other experiments.

To highlight the effect of the PC environment on metathase kinetics, we compared the catalytic activity with

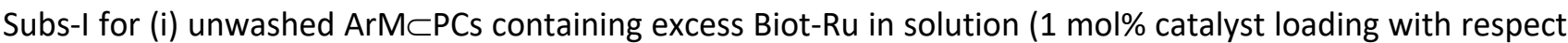




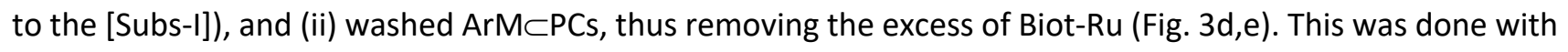
the most active mutant Sav ${ }^{\mathrm{N} 118 \mathrm{~K} \text { K121E }}$. According to ICP-MS, ca. 24\% of the added Biot-Ru catalyst is immobilized into the PC core (Supplementary Note 2 and Table S2). Interestingly, Subs-I affords only 8.8 TON $_{\text {Ru }}$ in (i), while $25 \mathrm{TON}_{\mathrm{Ru}}$ are detected after purification in (ii). Hence, the genetically-optimized ArMs are at least threefold more active within the PC's interior than the free cofactor Biot-Ru (Fig. 3e). We thus conclude that compartmentalization of the ArM in a crowded PC provides a propitious environment for catalysis.

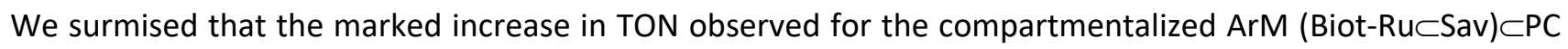

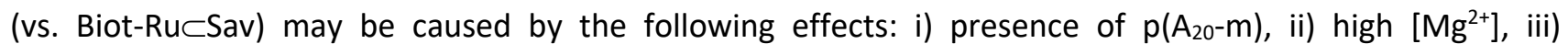
accumulation of Sub-I within the PCs or iv) crowding. Increasing the $\left[\mathrm{Mg}^{2+}\right]>200 \mathrm{mM}$ did not lead to a significant increase in TON (Supplementary Fig. S3c). HPLC analysis revealed a modest accumulation of Sub-I in empty PCs and in Sav-loaded PCs, Sav $\subset$ PCs $(<20 \%$, compared to the surrounding). Gratifyingly, addition of PEG $(3000,50 \mathrm{mg} / \mathrm{mL})$ led to a fivefold increase in TON vs. free Biot-RuCSav ${ }^{\mathrm{N} 118 \mathrm{~K} \mathrm{~K}^{121 \mathrm{E}}}$ for Subs-I, as well as for all other Sav isoforms. In stark contrast, the free co-factor Biot-Ru does not experience a change in the crowded environment (Fig. 3h). Accordingly, we hypothesize that the increased TON observed with (Biot$\left.\mathrm{Ru}_{\mathrm{S}} \mathrm{Sav}^{\mathrm{N} 118 \mathrm{~K} \mathrm{~K} 121 \mathrm{E}}\right) \subset \mathrm{PC}$ is primarily caused by crowding. ${ }^{37}$ Differences in the relative effect of crowding between

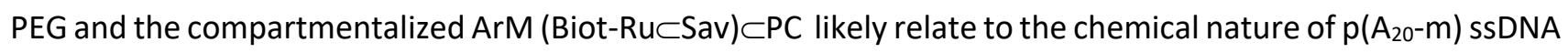
inside the PC. 
a ArM-catalyzed RCM (Subs-l) and uncaging umbelliferone inside the PCs

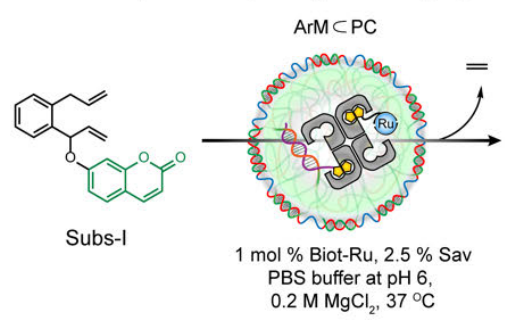

c Screening of Sav ${ }^{\text {mutant }}$ on RCM (Subs-I) inside the PCs

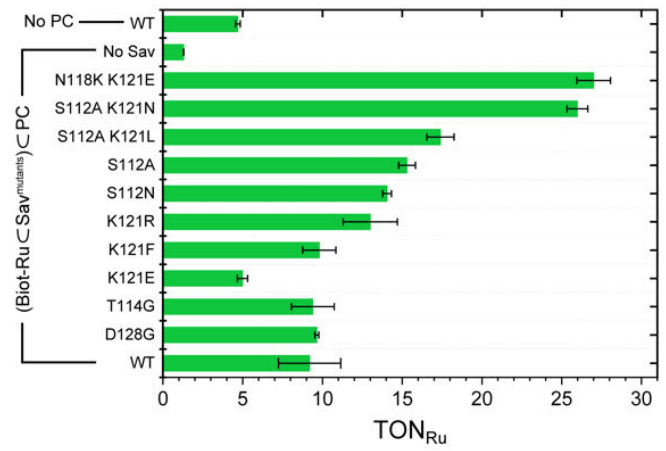

d Scenarios with free and bound Biot-Ru

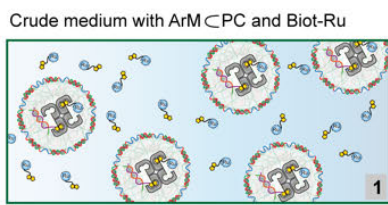

Washed medium with only ArM CPC

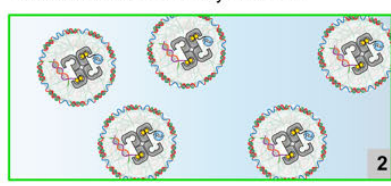

g Screening of Sav ${ }^{\text {mutant }}$ on RCM (Subs-II) inside the PCs

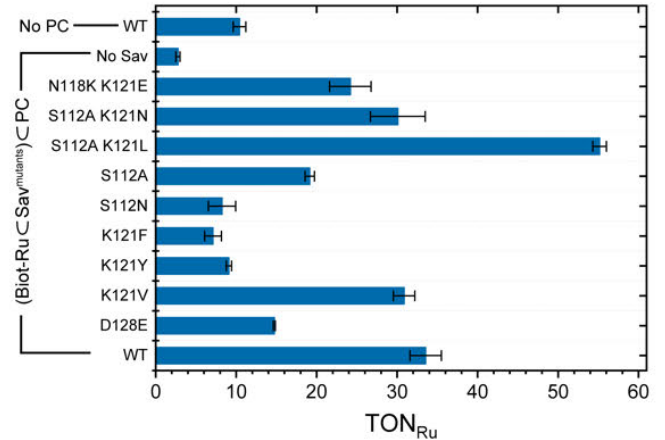

Artificial metathase: Biot-Ru $\subset$ Sav ${ }^{W T}$

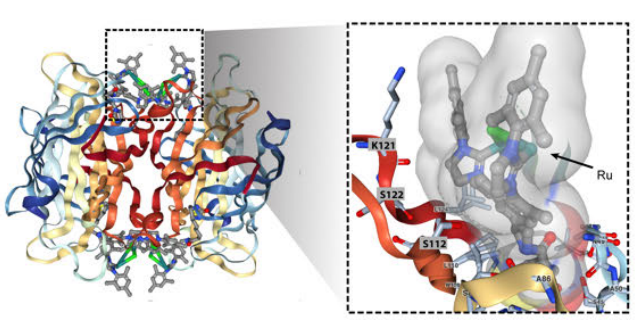

e ArM efficiency

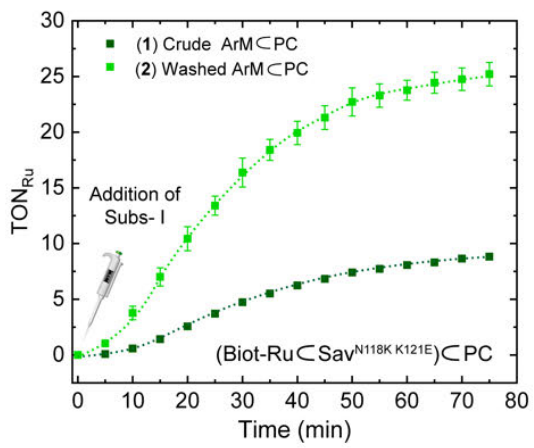

h Effect of molecular crowding on ArM (Subs-l)

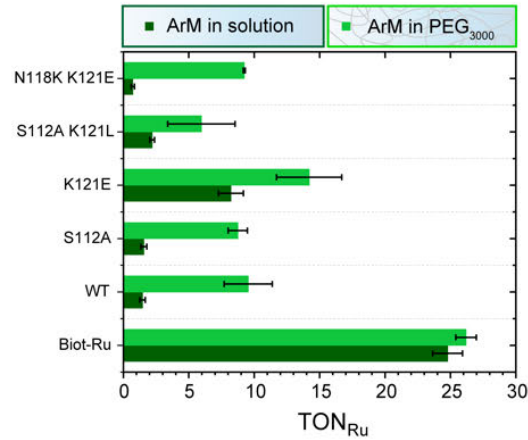

Fig. 3 | Intraprotocellular ring-closing metathesis (RCM) of Subs-I and Subs-II catalyzed by genetically-engineered ArMs. (a) Schematic representation of the RCM-triggered uncaging of umbelliferone inside the PCs from a caged precursor (Subs-I) (b) X-ray crystal structure (PDB: 5IRA) of the artificial metathase Biot-RuCSav ${ }^{\text {WT }}$. Biot-Ru and proximal protein residues are depicted as ball-and-stick and stick, respectively. (c) Genetic optimization of ArM for the uncaging of umbelliferone from Subs-I. The TON $\mathrm{Ru}_{\mathrm{u}}$ are based on yields of umbelliferone determined by fluorescence spectroscopy. Data are the means and standard deviation of duplicate reactions. (d) Schematic representation of two experiments: (1) $R C M$ is performed using a crude mixture of free Biot-Ru and ArM $\subset P C$, (2) RCM is performed using ArM $\subset P C$ after a washing step to remove unbound Biot-Ru (i.e., centrifugation followed by re-dispersion). The Ru content of these samples was determined by ICP-MS (Supplementary Table S2). (e) Comparison of the turnover number (TON $\mathrm{Ru}_{\mathrm{R}}$ ) of $A r M \subset P C$ vs. free Biot-Ru, revealing the effect of the environment resulting from compartmentalization within Sav $\subset$ PCs. (f) Schematic representation of the ArM-catalyzed RCM of a dimethoxynaphthalene precursor (Subs-II) inside PCs. (g) Genetic

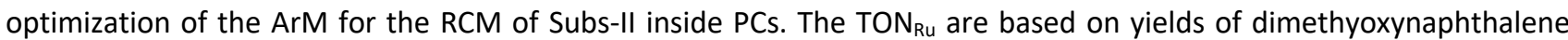
determined by GC-MS using an internal standard. Data are the means and standard deviation of duplicate reactions. ( $h$ ) Crowding effect on the TON $\mathrm{Ru}_{\mathrm{R}}$ resulting from addition of $50 \mathrm{mg} / \mathrm{mL}$ PEG (3000 Da) for different mutants, the WT and the free co-factor Biot-Ru.

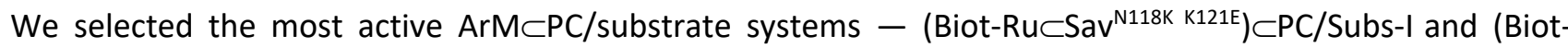

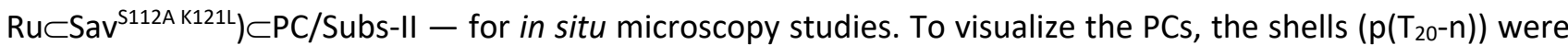
tagged with Atto $_{647}-\mathrm{n}^{*}$ (red channel, Fig. 4b) and the PCs were equipped with the ArM (details in SI). To monitor

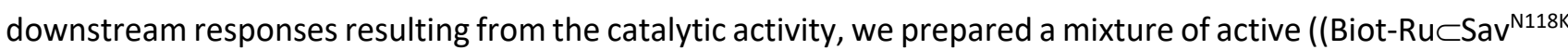
$\left.\left.{ }^{\mathrm{K} 121 \mathrm{E}}\right) \subset \mathrm{PC}\right)$ and dormant $\mathrm{PCs}\left(\mathrm{Sav}^{\mathrm{N} 118 \mathrm{~K} \mathrm{K121E}} \subset \mathrm{PC}\right)$. Both populations are initially indistinguishable with red shells and colorless cores (t=0 min, Fig. 4 a,b; Supplementary Fig. S5 shows start and end points for differently dyed 
PCs). Once Subs-I is injected, green fluorescence (excitation $405 \mathrm{~nm}$ ) appears in the core of the active PCs,

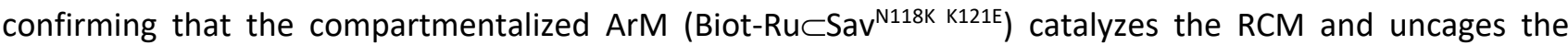
umbelliferone inside the PCs (Fig. 4b, Supplementary Fig. S4). The rate of umbelliferone release inside the active PCs (Fig. 4c; calculated from CLSM) correlates with the macroscopic fluorescence spectroscopy (Fig. 3e). Strikingly, a substantial expansion of the active PCs occurs during prolonged reaction (up to 5-fold in diameter and 125-fold in volume). The green fluorescence persists in the core and accumulates in the shell: it does not dilute visibly into the surrounding. This is indicative of tight binding to dsDNA, as most dsDNA is located in the shell and at the core/shell interface $\left(A_{20} / T_{20}\right.$ and $\left.A_{t t 0_{647}}-n * / n\right)$. Concomitantly, the red shell fluorescence decreases by about $50 \%$ after an hour of RCM activity. This is due to a thickness increase of the shell by swelling and potentially due to a loss of some Atto $_{647}-\mathrm{n} *$ by duplex breakage (see below). Since the PCs are under high osmotic pressure due to the entrapped $p\left(A_{20}-m\right)$ with all its counterions ${ }^{10}$ and only stabilized by the $A_{20} / T_{20}$ duplexes formed at the interface between the core and the shell, the swelling strongly suggests that the RCM products (i) intercalate into the dsDNA, thus (ii) weaken the dsDNA interactions substantially and lead to (iii) the dynamization of the membrane layer. Umbelliferone intercalates preferentially in A/T duplexes. ${ }^{35}$ The PC swelling caused by the release of dimethoxynaphtalene from Subs-II is less pronounced (3-fold vs. 5-fold increase in diameter, Fig. 4e,f,g; Supplementary Fig. S6). This is related to the poorer intercalating properties of dimethoxynaphthalene vs. umbelliferone. ${ }^{36}$ To further underscore the intercalation properties, Supplementary Fig. $S 7$ displays the melting curves of $A_{20}-m / T_{20}-m^{*}$ dsDNA in the presence of the intercalators. The melting curve flattens out in presence of umbelliferone, and the duplexes do not re-hybridize upon cooling, highlighting the dynamization of the duplexes. The perturbation of the duplex hysteresis is less pronounced for dimethoxynaphthalene.

Control experiments underscore the importance of the compartmentalization of the catalytic event within the PC for the produced metabolite to induce protocell growth. The expansion is not observed in the dormant PCs after the addition of Subs-I (Fig. 4d). This confirms that the Subs-I is not a morphogen, and that the metabolite (umbelliferone) produced in an active PC does not induce changes in a dormant PC via diffusion. Strikingly, in an additional control experiment, we added a high concentration of umbelliferone to dormant PCs and observed no morphological transformation, although the accumulation of umbelliferone (green fluorescence) within the shell and inside the core indicates its intercalation within the duplexes (Fig. 4h). Moreover, the morphology of dormant PCs remains unaltered upon addition of a mixture of both uncaged products, umbelliferone and naphthalene (Supplementary Fig. S8). These observations clearly demonstrate that the catalytic conversion inside the PCs is the decisive aspect to trigger downstream changes. Although unexpected, these observations highlight the possibility of emergent, self-inflicted behavior of active PCs. 
a RCM-induced protocell growth

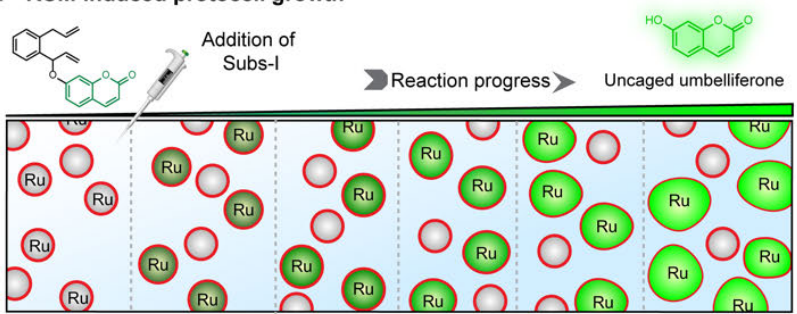

> $\left(\right.$ Sav $\left.^{\mathrm{N} 118 K \mathrm{~K} 121 \mathrm{E}}\right) \subset \mathrm{PC}$ (dormant)

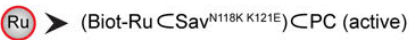

b Monitoring RCM (Subs-I) via CLSM imaging
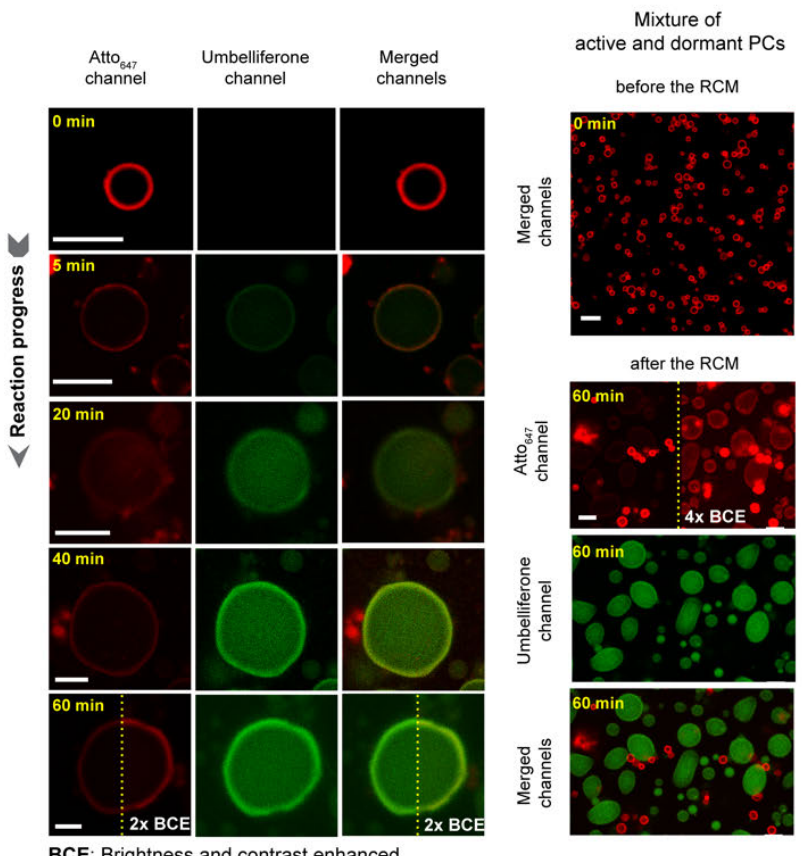

BCE: Brightness and contrast enhanced

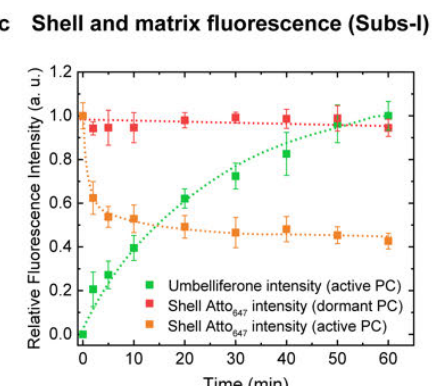

d Dimensional transformation of PCs

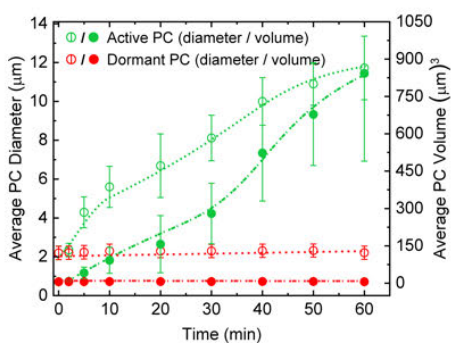

e Monitoring RCM (Subs-II) via CLSM imaging
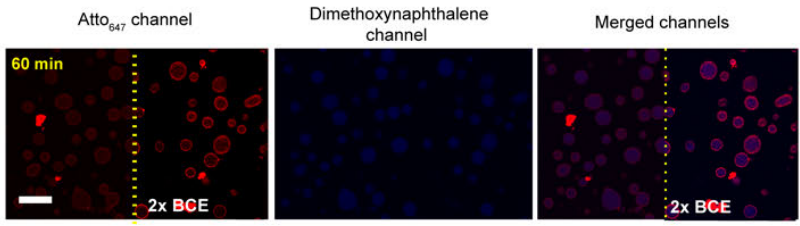

f Shell and matrix fluorescence (Subs-II) g Dimensional transformation of PCs
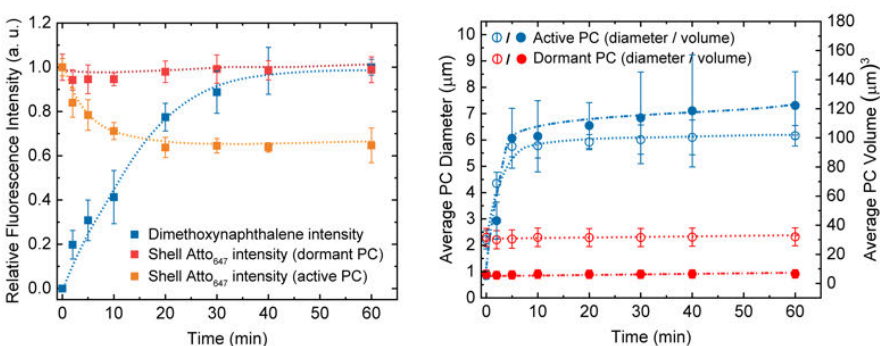

h Effect of uncaged product on the Sav-loaded PCs

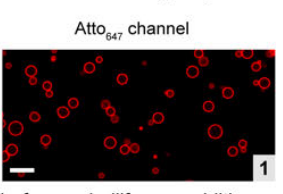

before umbelliferone addition

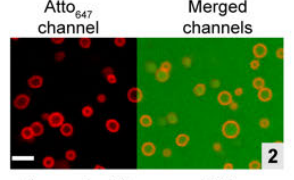

after umbelliferone addition

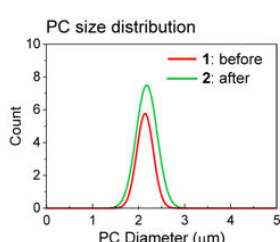

Fig. 4 | CLSM monitoring of ArM-catalyzed RCM inside PCs and ensuing morphological transformations. (a) RCM-

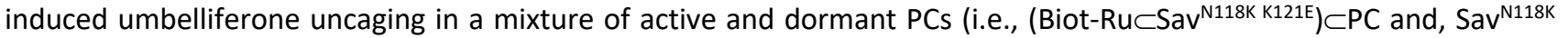
${ }^{\mathrm{K} 121 \mathrm{E}} \subset \mathrm{PC}$ ). Addition of Subs-I leads to umbelliferone accumulation within the active PCs, accompanied by a gradual increase in their volume. The dormant PCs remain unaltered, both in fluorescence and size. (b) Time-dependent CLSM images for RCM-induced umbelliferone uncaging in a single PC (left array) and a mixed PC system (right array). The shells of all PCs are labeled with Atto $647-n *$ (red channel). At $t=0$ (before the addition of Subs-I), all PCs are visible as red circles and indistinguishable. The RCM-induced uncaging of umbelliferone (green channel) is recorded over a 60 min period. The gradual increase in fluorescence intensity (green channel) in the core of the active PCs results from the RCM-induced uncaging of the umbelliferone. Brightness and contrast of some of the images (red and merged channels) are increased to visualize the shell of the active PCs. (c) Normalized time-dependent fluorescence intensities of umbelliferone (green trace), the shell of the active PCs (orange trace), and the shell of the dormant PCs (red trace). The fluorescence intensities are averaged from the CLSM images using 25 PCs. (d) Time-dependent increase in dimension for active and dormant PCs (averaged over 25 PCs). (e) CLSM images of the RCM-induced dimethoxynaphthalene formation inside the PCs. (f) Normalized time-dependent fluorescence intensities of the RCM product (blue trace), the shell of the active PCs (orange trace), and the shell of the dormant PCs (red trace). (g) Time-dependent RCM-induced change in the dimensions for active and dormant PCs (averaged over 25 PCs). The dimethoxynaphthalene product has less influence on the morphological

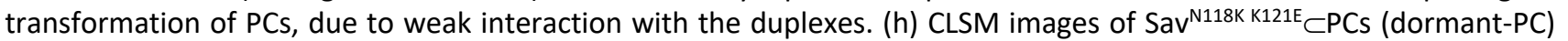
before and after the addition of umbelliferone $(500 \mu \mathrm{M})$. The size distribution (shown on the right) indicates that the morphology of the PCs remains unaltered when the intercalator was added in solution even though the diffusion of umbelliferone is observed into the PC core. Scale bar: $5 \mu \mathrm{m}$. Conditions: $1 \%$ catalyst loading, $100-300 \mu \mathrm{M}$ of substrates, $10 \mathrm{mM}$ phosphate buffer, $\mathrm{pH}$ 6, $0.1 \mathrm{M} \mathrm{MgCl}_{2}$.

From above, it is evident that the released umbelliferone from Subs-I is a more powerful morphogen compared to dimethoxynaphthalene released from Subs-II. Next, we showcase signal-transduction cascades - a distinctive feature of living systems - that result from the compartmentalized catalytic event using Subs-I. 
Therein, the release of the abiotic metabolite produced from Subs-I is exploited to drive the metamorphosis of the PCs, leading to distinct functional intraparticle adaptation, and ultimately, interparticle interaction.

First, we surmised that the swelling and internal stress may be harnessed to break specifically engineered dsDNA junctions, allowing to realize a color change. To this end, we embedded a mechano-fluorescent forcesensing module (FM) as a crosslinker within an active PC by hybridization with the $\mathrm{m}$ barcodes (Fig. 5a, Supplementary Fig. S9a,b). ${ }^{12}$ The FM consists of a short dsDNA arranged in a zipper geometry and equipped with a fluorophore (Cy5, yellow-red dot) and a quencher (red-black dot, Fig. 5a). The dsDNA zipper is designed to be the weakest network link $\left(-\Delta \mathrm{G}_{\mathrm{y} / \mathrm{y}^{*}}=25.8 \mathrm{kcal} \mathrm{mol}^{-1}>-\Delta \mathrm{G}_{\mathrm{z} / \mathrm{z}^{*}}=25.2 \mathrm{kcal} \mathrm{mol}^{-1}>-\Delta \mathrm{G}_{\mathrm{m} / \mathrm{m}^{*}}=24 \mathrm{kcal} \mathrm{mol}^{-1}>\right.$ $\left.-\Delta G_{x / x^{*}}=9 \mathrm{kcal} \mathrm{mol}^{-1}\right)$. Its zipper geometry compared to normal dsDNA facilitates mechanical opening. The homogeneous loading of such FMs was confirmed by functionalization with a FM without the quencher (Supplementary Figure S10). Upon addition of Subs-I, the catalytic production of umbelliferone leads to swelling and the mechanical stress induces the rupture of the FM, as revealed by increasing fluorescence resulting from the Cy5 reporter (Fig. 5c, Supplementary Fig. S9c). The PCs acquire a new red fluorescence color. In line with the signal-transduction cascade mechanism, a delay (ca. $20 \mathrm{~min}$ ) in the appearance of the Cy5 fluorescence compared to the primary fluorescence caused by umbelliferone uncaging is observed (Supplementary Fig. S9e). This reflects the critical stretching threshold that the FM requires for unzipping. Correspondingly, the signal transduction cascade allows for functional adaptation revealed by the appearance of a new color (a simple PC phenotype) and leads to the exposure of two previously masked ssDNA sequences that become available for further DNA-based reaction schemes.

Building on the observed growth, we hypothesized that higher substrate concentrations and extended reaction times may lead to shell rupture and promote PC interactions. Indeed, when subjecting the active PCs to 3-fold Sub-I concentration (1 mM), fusion of PCs occurs. These fusion events are triggered by spontaneous, growth-induced symmetry-breaking of the original spherical PCs (most likely due to defects in the shell) and ultimately shell rupture, whereupon interaction between the core material occurs (Fig. $5 \mathrm{~d}, \mathrm{e}$ ). The symmetry breaking is clearly evidenced by the observation of highly fluorescent hemispherical caps distant from the fusion center. A time-lapse fusion event is shown in Fig. $3 f$ (see also Supplementary Fig. S11). Since the interior is gelled due to the presence of the tetravalent Sav, a complete secretion of the interior as liquid phase cannot occur. We suggest that the fusion into rather homogeneous assemblies is assisted by (i) gaining additional inter-protocellular biotin-Sav interactions and (ii) minimizing the interface with the water. 
a Mechanofluorescent reporter

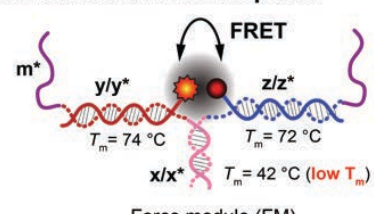

b

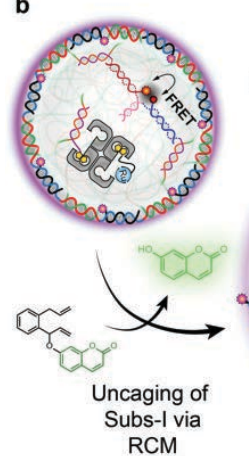

c RCM-induced self-reporting protocellular adaptation

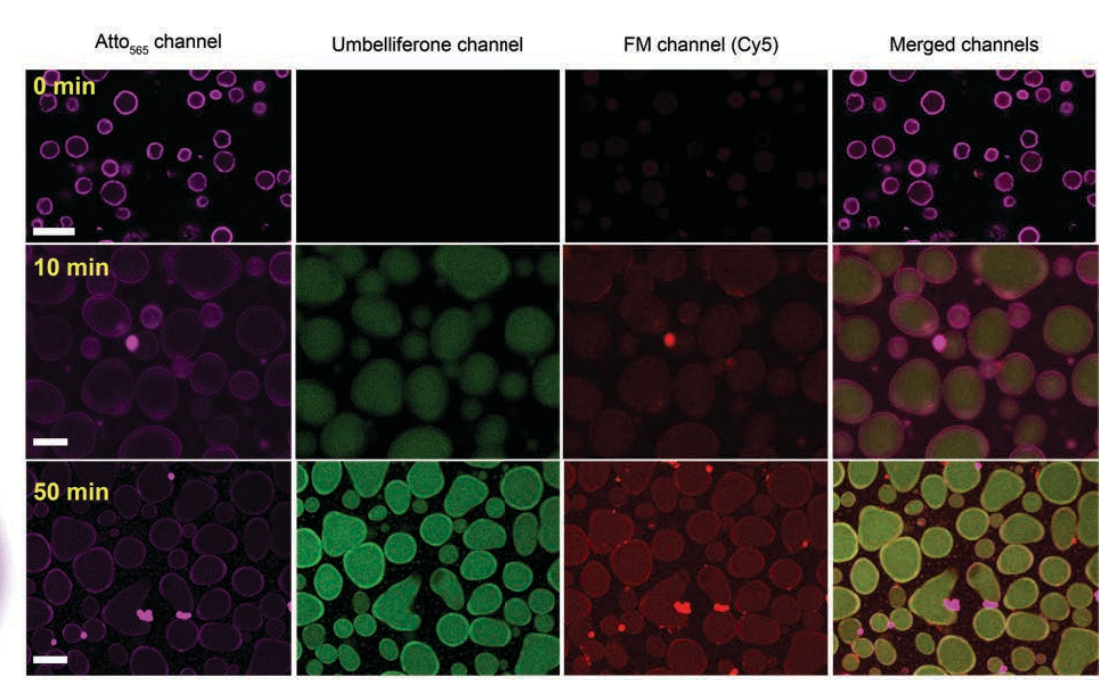

RCM-induced membrane dynamization and protocell fusion

d Umbelliferone-induced PC growth and fusion

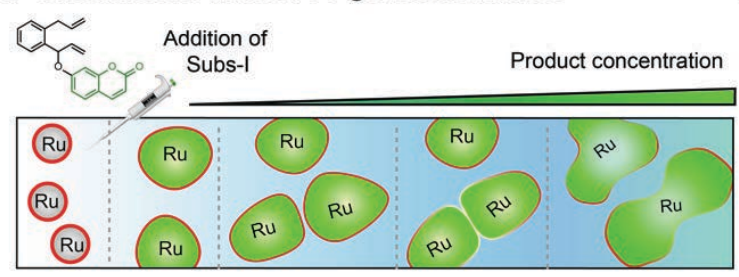

f Time-lapse CLSM images of a fusion event

$\mathrm{PC}$ swelling $\rightarrow$ membrane softening $\rightarrow$ interdigitation $\rightarrow$ fusion

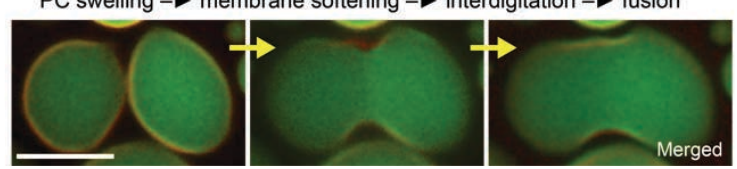

e CLSM imaging of morphological transformations

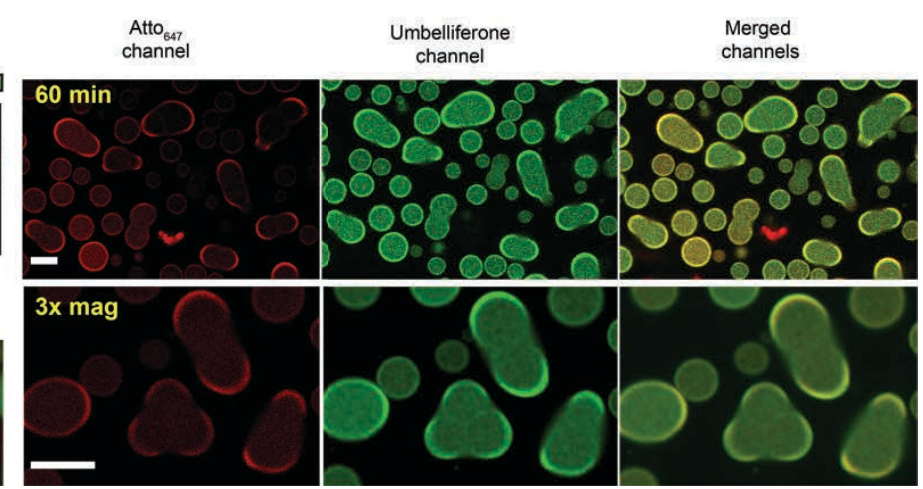

Fig. 5 | RCM-induced, self-reporting, downstream functional adaptation and morphological output. (a) Structure of the mechanofluorescent force-sensing module (FM). The red-emitting fluorophore (Cy5) and the quencher (Iowa RQ) are in close proximity, thus quenching the red fluorescence by FRET. The FM has two $\mathrm{m}^{*}$ domains, which are used to crosslink

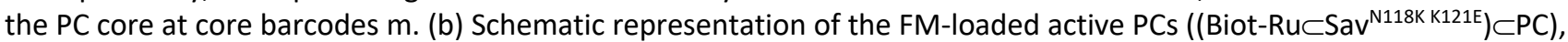
in which $70 \%$ of the barcodes $(\mathrm{m})$ inside the PCs are used for catalyst loading and $30 \%$ are used to attach the FM. RCMinduced uncaging of umbelliferone triggers PC swelling and FM activation as revealed by red fluorescence. (c) Timedependent CLSM. The magenta channel represents the Atto $565-n$ * conjugated PC shells; the green channel reports on the uncaging of umbelliferone inside the PC core, and the red channel reveals the second fluorescent output from the dissociated FM. The isolated fluorescent "hotspots" are contamination, while the PC interior turns homogeneously red. (d) Schematic representation of RCM-induced morphological transformation in PCs. The PCs undergo swelling and membrane rupture and PC fusion upon increasing the concentration of the Subs-I. (e) CLSM images of swollen and fused PCs after 60 min following Sub-I addition. (f) Time-lapse fusion event of protocells. Larger overview in Supplementary Fig. S11. Scale bars: $5 \mu \mathrm{m}$. 3x mag: three times magnified.

In summary, we introduced a generic strategy to encapsulate artificial metalloenzymes (ArMs) inside the macromolecularly crowded core of all-DNA PCs. We then highlighted how 'abiotic' catalytic signal conversion strategies can be used for downstream morphological adaptation at varying levels of complexity. The key step relies on selecting a synthetic, non-DNA substrate that can be turned into a metabolite able to interact and weaken DNA duplexes. This activates the swelling of the PCs trapped in a metastable state (being under high osmotic pressure), resulting in (i) PC growth, (ii) activation of designed mechanophores for functional adaptation (e.g. color), and (iii) PC fusion. Such features are reminiscent of the behavior of living cells - albeit 
on simplistic levels. We unraveled that the ArMs are more active inside the crowded environment and that site-directed mutagenesis of the ArMs improves the catalytic performance. Remarkably, all morphological changes in the PCs are observed only when performing catalysis inside the PCs: The external addition of substrates or products to inactive PCs does not induce any morphological change.

These findings reveal the opportunities of endowing PCs with abiotic catalytic activity, here 'abiotic' in the sense of being foreign to typical DNA-systems, to generate chemical signals compartmentalized within hybrid protocellular environments. These PCs display advanced adaptive and emergent behavior. While we highlighted pathways towards downstream functions on a morphological level, we believe that the merger of artificial metalloenzymes and colloidal coacervates can provide avenues for adaptive functional systems in sensor applications or as colloidal factories for functional compounds. Although cross-disciplinary approaches to explore the design, structuration, function and evolutionary potential of metabolic PCs with genetically evolved proteinaceous catalysts is in its infancy, our approach provides valuable insights (i) into the acquisition of chemically-triggered adaptive behavior of prebiotic coacervates and (ii) towards a minimalistic design of life-like abiotic systems. Capitalizing on the interaction between dsDNA and abiotic metabolites presented herein, we envision fascinating opportunities to further engineer interactive PC systems, that can communicate, translate and process various types of signals in more complex sensory environments.

\section{Supplementary Information}

Supplementary information is available in the online version of the paper. Reprints and permission information is available online at www.nature.com/reprints. Correspondence and requests for materials should be addressed to A. Walther and T. R. Ward.

\section{Author Contributions}

A.S. and A.W. conceived the project. A.S., V.S., A. W. and T. R. W. designed and performed all the experiments. A.S. analyzed the data and prepared the preliminary draft. A.W., T.R.W supervised the project. All authors contributed to writing the manuscript.

\section{Acknowledgments}

We acknowledge support by the European Research Council Starting grant to AW (TimeProSAMat, (agreement 677960) and Advanced Grant to TRW (DrEAM, agreement 694424), the DFG Cluster of Excellence livMatS "Living, Adaptive and Energy-Autonomous Materials Systems" and the NCCR Molecular Systems Engineering. A.S. acknowledges the support by the Alexander von Humboldt Foundation. 


\section{Competing Financial Interests statement}

The authors declare no competing financial interests.

\section{Data Availability Statement}

The data that support the plots within this paper and other finding of this study are available from the corresponding author upon reasonable request.

\section{Reference}

$1 \quad$ Oparin, A. I. Origin of Life (Dover, 1952).

2 Haldane, J. B. S. The origin of life. Ration. Annu. 148, 3-10 (1929).

3 Brangwynne, C. P. et al. Germline P granules are liquid droplets that localize by controlled dissolution/condensation. Science 324, 1729-1732 (2009).

4 Pawson, T. Specificity in signal transduction: from phosphotyrosine-SH2 domain interactions to complex cellular systems. Cell 116, 191-203 (2004).

5 Pawson, T. \& Nash, P. Assembly of cell regulatory systems through protein interaction domains. Science 300, 445-452 (2003).

6 You, L., Cox, R. S., Weiss, R. \& Arnold, F. H. Programmed population control by cell-cell communication and regulated killing. Nature 428, 868-871 (2004).

7 Bacchus, W. et al. Synthetic two-way communication between mammalian cells. Nat. Biotechnol. 30, 991-996 (2012).

$8 \quad \mathrm{Na}$, S. et al. Rapid signal transduction in living cells is a unique feature of mechanotransduction. Proc. Natl. Acad. Sci. U.S.A. 105, 6626-6631 (2008).

9 Trepat, X. et al. Universal physical responses to stretch in the living cell. Nature 447, 592-595 (2007).

10 Merindol, R., Loescher, S., Samanta, A. \& Walther, A. Pathway-controlled formation of mesostructured all-DNA colloids and superstructures. Nat. Nanotechnol. 13, 730-738 (2018).

11 Jeschek, M. et al. Directed evolution of artificial metalloenzymes for in vivo metathesis. Nature 537, 661-665 (2016).

12 Merindol, R., Delechiave, G., Heinen, L., Catalani, L. H. \& Walther, A. Modular design of programmable mechanofluorescent DNA hydrogels. Nat. Commun. 10, 528 (2019).

13 Changeux, J.-P. \& Christopoulos, A. Allosteric Modulation as a Unifying Mechanism for Receptor Function and Regulation. Cell 166, 1084-1102 (2016).

14 Martin, N. Dynamic synthetic cells based on liquid-liquid phase separation. ChemBioChem 20, 25532568 (2019).

15 Sokolova, E. et al. Enhanced transcription rates in membrane-free protocells formed by coacervation of cell lysate. Proc. Natl. Acad. Sci. U.S.A. 110, 11692-11697 (2013).

16 Zwicker, D., Seyboldt, R., Weber, C. A., Hyman, A. A. \& Jülicher, F. Growth and division of active droplets provides a model for protocells. Nat. Phys. 13, 408-413 (2017).

17 Mansy, S. S. et al. Template-directed synthesis of a genetic polymer in a model protocell. Nature 454, 122 (2008).

18 Adamala, K. \& Szostak, J. W. Nonenzymatic template-directed RNA synthesis inside model protocells. Science 342, 1098-1100 (2013).

19 Adamala, K. \& Szostak, J. W. Competition between model protocells driven by an encapsulated catalyst. Nat. Chem. 5, 495-501 (2013).

20 Langton, M. J., Scriven, L. M., Williams, N. H. \& Hunter, C. A. Triggered release from lipid bilayer vesicles by an artificial transmembrane signal transduction system. J. Am. Chem. Soc. 139, 1576815773 (2017).

21 Fulton, A. B. How crowded is the cytoplasm? Cell 30, 345-347 (1982). 

compartmentalization. Nat. Chem. 4, 941-946 (2012).

23 Crosby, J. et al. Stabilization and enhanced reactivity of actinorhodin polyketide synthase minimal complex in polymer-nucleotide coacervate droplets. Chem. Commun. 48, 11832-11834 (2012).

Booth, R., Qiao, Y., Li, M. \& Mann, S. Spatial positioning and chemical coupling in coacervate-inproteinosome protocells. Angew. Chem. Int. Ed. 58, 9120-9124 (2019).

Gobbo, P. et al. Programmed assembly of synthetic protocells into thermoresponsive prototissues. Nat. Mater. 17, 1145-1153 (2018).

Joesaar, A. et al. DNA-based communication in populations of synthetic protocells. Nat. Nanotechnol. 14, 369-378 (2019).

Walther, A. Viewpoint: From Responsive to Adaptive and Interactive Materials and Materials Systems: A Roadmap. Adv. Mater., 1905111 (2019).

Wilson, M. E. \& Whitesides, G. M. Conversion of a protein to a homogeneous asymmetric hydrogenation catalyst by site-specific modification with a diphosphinerhodium(I) moiety. J. Am. Chem. Soc. 100, 306-307 (1978).

Schwizer, F. et al. Artificial metalloenzymes: reaction scope and optimization strategies. Chem. Rev. 118, 142-231 (2018).

Okamoto, Y. et al. A cell-penetrating artificial metalloenzyme regulates a gene switch in a designer mammalian cell. Nat. Commun. 9, 1943 (2018).

$\mathrm{Ji}, \mathrm{X}$. et al. Click and release: bioorthogonal approaches to "on-demand" activation of prodrugs. Chem. Soc. Rev. 48, 1077-1094 (2019).

Fan, X. et al. Optimized tetrazine derivatives for rapid bioorthogonal decaging in living cells. Angew. Chem. Int. Ed. 55, 14046-14050 (2016).

33 Sabatino, V., Rebelein, J. G. \& Ward, T. R. "Close-to-release": spontaneous bioorthogonal uncaging resulting from ring-closing metathesis. J. Am. Chem. Soc. 141, 17048-17052 (2019).

34 Wilner, O. I. et al. Enzyme cascades activated on topologically programmed DNA scaffolds. Nat. Nanotechnol. 4, 249-254 (2009).

35 Ou, C.-N., Tsai, C.-H., Tapley, K. J. \& Song, P.-S. Photobinding of 8-methoxypsoralen and 5,7dimethoxycoumarin to DNA and its effect on template activity. Biochemistry 17, 1047-1053 (1978).

36 Banks, T. M., Clay, S. F., Glover, S. A. \& Schumacher, R. R. Mutagenicity of N-acyloxy-N-alkoxyamides as an indicator of DNA intercalation part 1: evidence for naphthalene as a DNA intercalator. Org. Biomol. Chem. 14, 3699-3714 (2016).

37 Ellis, R. J. Macromolecular crowding: obvious but underappreciated. Trends Biochem Sci. 26, 597-604 (2001). 